\title{
TGRID LOCATION SERVICE IN AD-HOC NETWORKS
}

\author{
Baktash Motlagh Farrokhlegha \\ Department of Computer, Technical\& vocational University, Urmia, West \\ Azarbayjan, IRAN
}

\begin{abstract}
Geographic addresses are essential in position-based routing algorithms in mobile ad hoc networks, i.e. a node that intends to send a packet to some target node, has to know the target's current position. A distributed location service is required to provide each node's position to the other network nodes. Hierarchical Location Service (HGRID) has been known as a promising location service approach. In this paper we present a new approach called TGRID and describe the performance of a novel multi-level Tree-walk grid location management protocol for large scale ad hoc networks. The Tree-walk grid location service mechanism is evaluated by GLOMOSIM against well known location service protocol HGRID when increasing node density and node speed. It is observed that TGRID outperforms HGRID in terms of packet delivery fraction and storage cost and also maintains low control overhead in a uniformly randomly distributed network.
\end{abstract}

\section{KEYWORDS}

Location based routing, location service, location management, Mobile Ad Hoc Networks, HGRID, and TGRID

\section{INTRODUCTION}

Routing in a mobile ad hoc network is a demanding task since the network's topology is changing frequently. In early approaches, proactive and reactive routing protocols were suggested [6][19]. These protocols maintain information about paths in a network. This may be difficult with frequent topology changes. Position-based routing algorithms make routing decisions based only on the position of the communication partners, and offer a scalable solution to this problem [5][12]. Different position-based routing protocols have been developed for ad hoc networks. Some of these protocols assume that at least a location service exists which provides location information of all the mobile nodes in the network. Hierarchical Location Service (HGRID) is one of the methods for providing location information in an ad hoc network [1][14]. In HGRID, each mobile node periodically informs a set of location servers with its current location when it moves to new location. The set of location servers chosen is determined by a predefined geographic grid[19].

HGRID is a distributed location service which tracks mobile node locations. HGRID combined with geographic forwarding allows the construction of ad hoc mobile networks that scale to a larger number of nodes than possible with the previous works. In this work, we propose an ad-hoc mobility management scheme. Routing is carried out in the hierarchical network structure. The location of a mobile host is defined in terms of the positional relationships between the mobile host and location server zone. In particular, we will define the location of a mobile host as the ID number of its nearest location database zone. The nodes containing the location databases can dynamically detach and re-attach to the network at any time due to mobile nodes' movement or 
changes in the communications environment [4][11][17]. However, the temporally interruption in a node's connectivity to the network should have minimum effect on the other nodes' communication. This imposes great challenges in the design and operation of an ad-hoc network [4]. This paper proposes TGRID distributed location service algorithm and compares its scalability with HGRID (one of the best location services)[18].

The rest of the paper is as following. In section 2 we overview HGRID algorithm. Section 3 describes the implementation of the proposed algorithm (TGRID) in detail. Section 4 analyses HGRID and TGRID performance and scalability using GLOMOSIM [8][15]. Section 5 summarises the paper's contributions and compares our scheme with one of the well known schemes in location management. It also concludes the paper and suggests areas for future improvements.

\section{HGRID LOCATION SERVICE}

HGRID defines a hierarchy of $\mathrm{K}$ levels $(\mathrm{L} 1 \ldots \mathrm{LK})$ on unit grid regions. Each $\mathrm{Li}+1$ quadrant is composed of four Li quadrants. At each level, the leader selection is as follows: for level I $(1 \leq \mathrm{i} \leq$ $\mathrm{k}-1$ ), the top rightmost Li-1 leader is the i-th hierarchical leader of the bottom left Li grid, top leftmost Li-1 leader is the hierarchical leader of the bottom right Li grid, bottom rightmost Li-1 leader is the hierarchical leader of the top left Li grid, and the bottom leftmost Li-1 leader is the hierarchical leader of the top right Li grid. The top of the hierarchy, (LK), is defined by the four Lk-1 grids. A node that is physically located in an i-th hierarchical grid is responsible for the duty of an i-th hierarchical location server [3][13].

When u moves across two Order-1 regions, if the movement is within the region under the same L1 leader, $\mathrm{u}$ sends a location update to L1. Otherwise, $\mathrm{u}$ additionally sends an update to its previous L1 grid indicating its departure. Further, if the movement specified in the update requires the next hierarchical server to be notified, it forwards the packet to that grid.

To discover a node u's location, $v$ sends a query packet to u's L1 leader. If $u$ and $v$ are in the same i-th grid, the query has to be forwarded until it reaches an i-th server (in the worst case), before a location reply can send back. Since the location databases in the upper levels of the hierarchy carry the approximate location information of nodes, location replies from these servers return the address of the server who has more accurate information of $u$ [7].

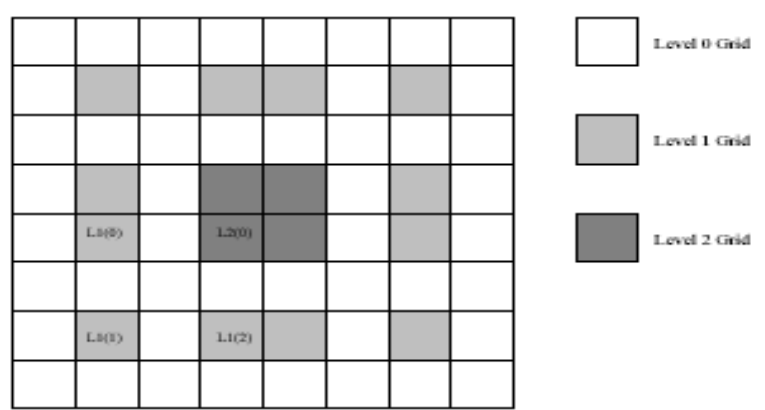

Figure 1. various levels in HGRID [7]

\section{HGRID}

TGRID Location Service divides the area containing the ad hoc nodes into a hierarchical grid of squares As GLS [1]. 
TGRID combines $9^{i}$ order- 1 grid to form an order-i Grid $(0 \leq \mathrm{i} \leq \mathrm{H})$ where $\mathrm{H}$ is the highest level number. Each node selects the middle square in each order i region as its location server zone (Home Region). The structure of TGRID is shown in Figure 2. For a node C to be node A's location server, $\mathrm{C}$ must be in the middle square of nine grids in level i grid where A resides. The location server regions store multi-grained information, i.e., each level-i location server zone stores the information of which Level-(i-1) zone the node is in, and only level-1 location servers store the exact location of A. Each node updates its Level-1 location server zone with its exact location and updates higher level location server zone with its relative location. If the node-B needs node-A's geographic location, it sends the query to its own level-1 location server zone. If the nodes in that zone have any information about A's location, they send the answer to B, otherwise the request forwarded to higher level location server zone until it reaches the last zone (most central square in the whole area). When B gets the answer, and it's the relative location of $\mathrm{A}$, the query is recursively forwarded to the closer location server zones to A until it reach the one that know the exact location of A. one of the nodes in this zone sends back the answer to B. Location Discovery and Data Transfer in TGRID is shown in figure 3.

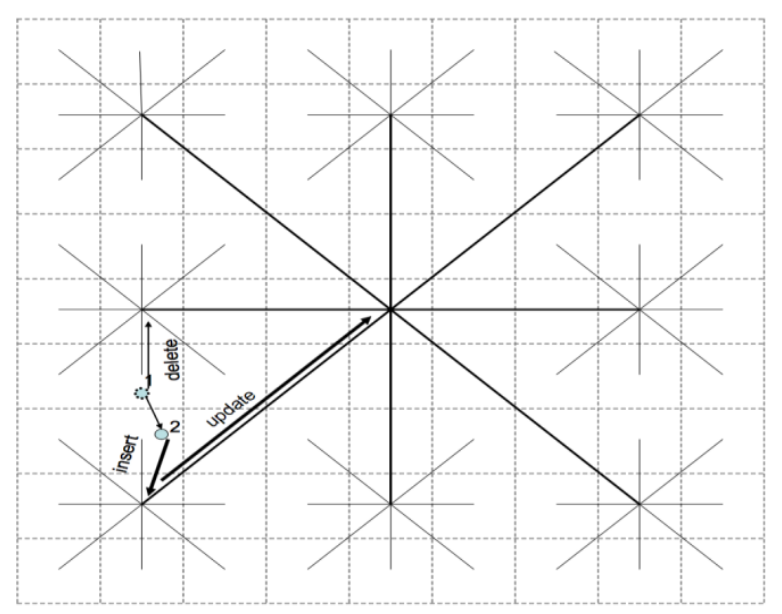

Figure 2. location update

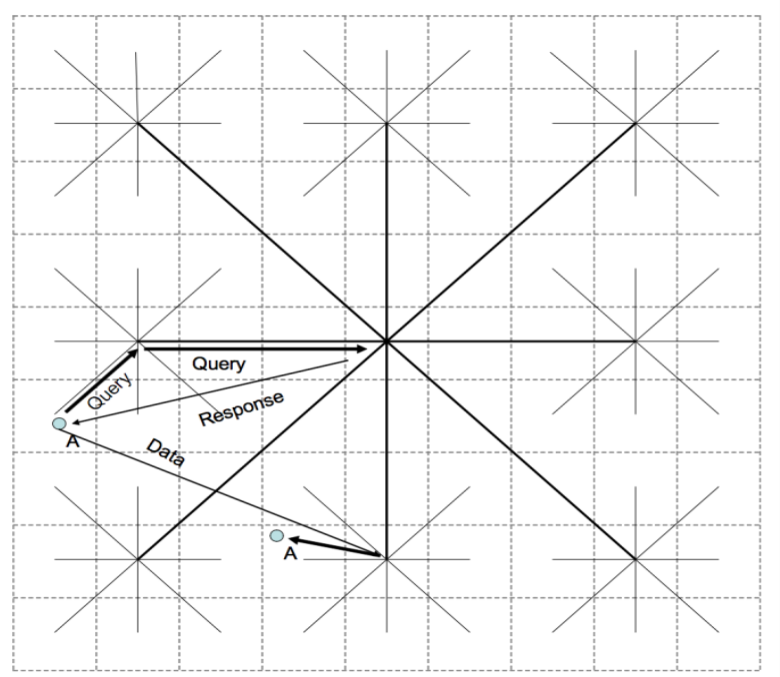

Figure 3. Location Discovery And Data Transfer 


\section{Performance Studies}

We implemented the TGRID protocol as well as HGRID [7] in Glomosim [8] as separate location management layers that operate in conjunction with IP.

Location table and neighbour table are two Main data structures in the location management layer. If the location of the destination is unknown, Data from transport layer is queued in a buffer and a location query is sent to the destination's location server. Packet lifetime in the buffer is 4 seconds. Periodic broadcast protocol enables each node to realise its local connectivity, and records it in the neighbour table to assist in geographic routing. MFR[9] without backward progression, in which packets are dropped if no forward progress can be made, was implemented as the geographic routing algorithm[16].

The first study fixed the simulation area to be $6750 \times 6750 \mathrm{~km} 2$ and the average node speed to be 5 $\mathrm{m} / \mathrm{sec}$ while varying the total number of nodes in the network. In the second study simulation area was fixed to $2250 \times 2250 \mathrm{~km} 2$ consisting of 320 nodes by varying the average node speed. Specific parameters for our simulations are listed in table I.

TABle 1. Simulation Parameters

\begin{tabular}{|c|c|c|}
\hline Parameters & Scenario I & Scenario II \\
\hline Simulation Time & $300 \mathrm{~s}$ & $300 \mathrm{~s}$ \\
\hline Simulation Area & $6750 \times 6750 \mathrm{~m}^{2}$ & $2250 \times 2250 \mathrm{~m}^{2}$ \\
\hline Unit Grid Size & $250 \mathrm{~m} / \mathrm{s}$ & $250 \mathrm{~m} / \mathrm{s}$ \\
\hline Number of Nodes & $720-2280$ & 320 \\
\hline $\begin{array}{c}\text { Transmission } \\
\text { Range }\end{array}$ & $350 \mathrm{~m}$ & $350 \mathrm{~m}$ \\
\hline $\begin{array}{l}\text { Transmission } \\
\text { Speed }\end{array}$ & $54 \mathrm{~m} / \mathrm{s}$ & $54 \mathrm{~m} / \mathrm{s}$ \\
\hline MAC Protocol & IEEE 802.11 & IEEE 802.11 \\
\hline Mobility Model & RandomWaypoint & Random Waypoint \\
\hline Maximum Speed & $10 \mathrm{~m} / \mathrm{s}$ & $0-25 \mathrm{~m} / \mathrm{s}$ \\
\hline CBR Rate & 1 Packet & 2 Packet/s \\
\hline Pause Time & 0 & 0 \\
\hline Traffic Pattern & Random & Random \\
\hline Buffer Size & 1000 packets & 1000 packets \\
\hline Data Payload & 512 bits & 512 bits \\
\hline CBR connections & 1000 & 160 \\
\hline
\end{tabular}

To test the efficiency of the protocols for location discovery as well as efficient delivery of data, we initialised 1000 CBR connections, where the source and destination nodes are chosen randomly for all the scenarios. Each connection sends one data packet, randomly starts after 150 seconds into the simulation and terminates randomly at 250 seconds into the simulation. 


\subsection{Simulation Results}

Figures 4 and 5 indicate the fraction of data packets successfully delivered by each protocol and the query success ratio. TGRID performs almost better than HGRID but in most of cases both protocol performs the same.

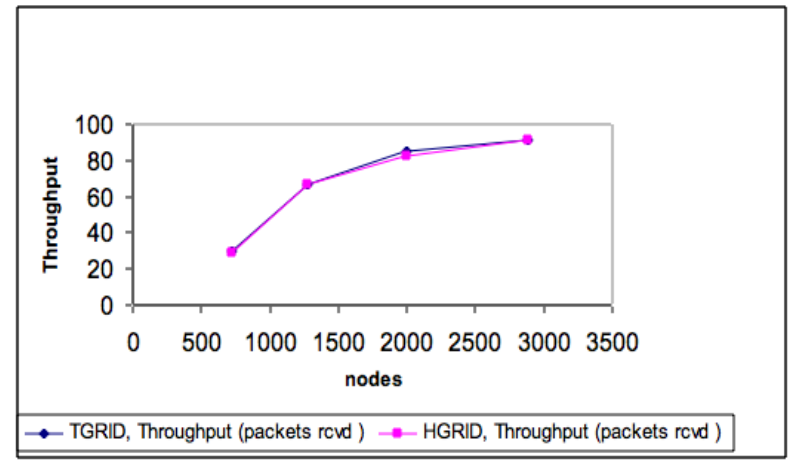

Figure 4. Data Throughput

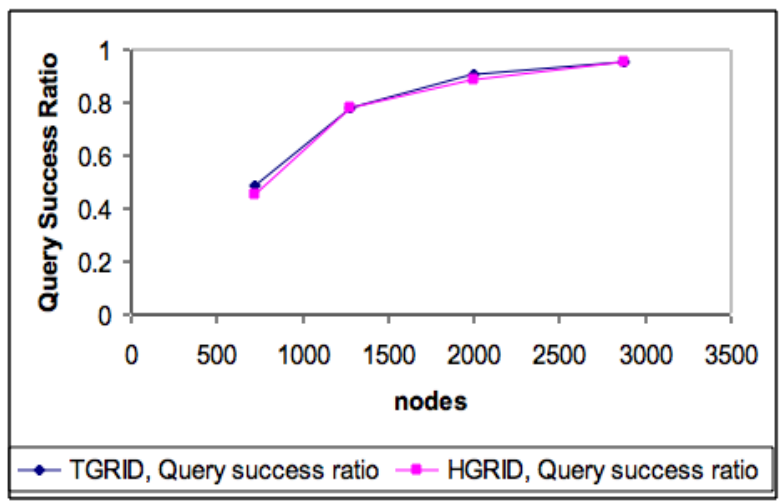

Figure 5.Query Success Ratio

Figure 6 and 7 shows the average control overhead for both protocols. However for both protocols the update overhead increases only logarithmically with the number of nodes, TGRID has less control overhead than HGRID. As shown in figure 6 with the packet overhead being nearly equal for both protocols, the average number of bytes being transmitted is more for HGRID (figure 7).

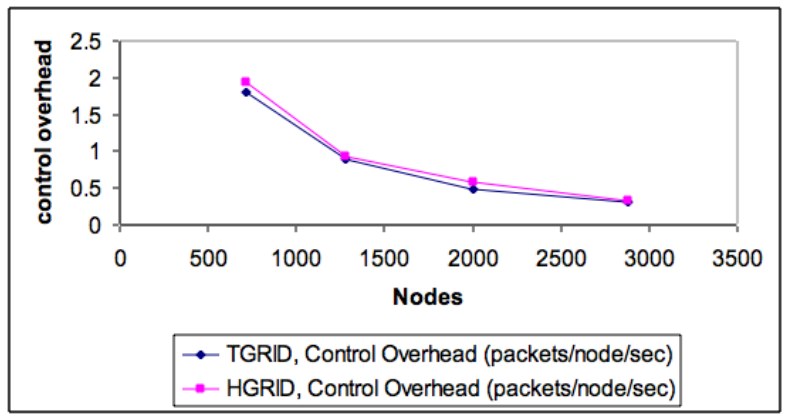

Figure 6.Control Overhead 


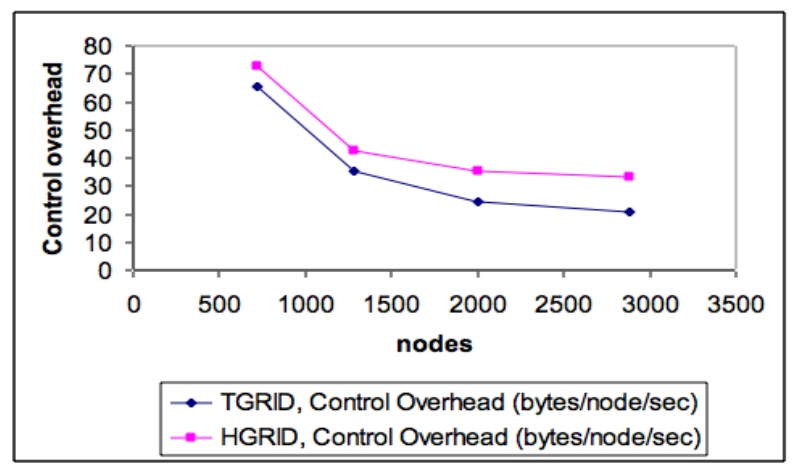

Figure 7.Control Overhead

Finally, figure 8 shows the increase in location database size of both protocols with increase in number of nodes. TGRID has less memory requirements than HGRID, since number of location databases are less.

However, in both protocols the average of database size grows only slightly with the increase in network size and both protocols are scalable.

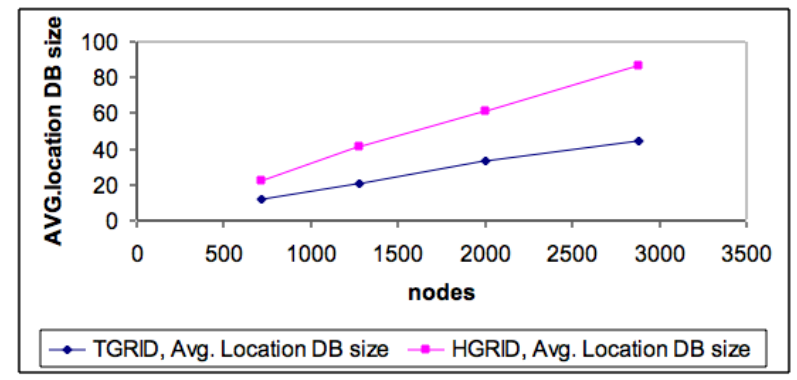

Figure 8.Location Database Size

Previous figures show the effect of node density on location management and how different location management protocols affect the performance of geographic routing.

We also considered the effect of mobility on performance of the network and varied the maximum speed in the Random Waypoint model to change the average mobility of the nodes. An increase in mobility is proportional to the rate at which nodes cross grid boundaries and new updates sent out to location servers. Each plot point presented here is an average of seven simulation runs.

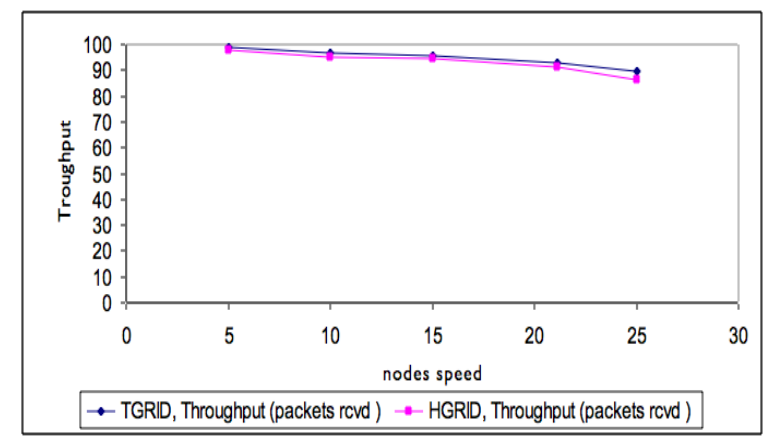

Figure 9.Throughput 


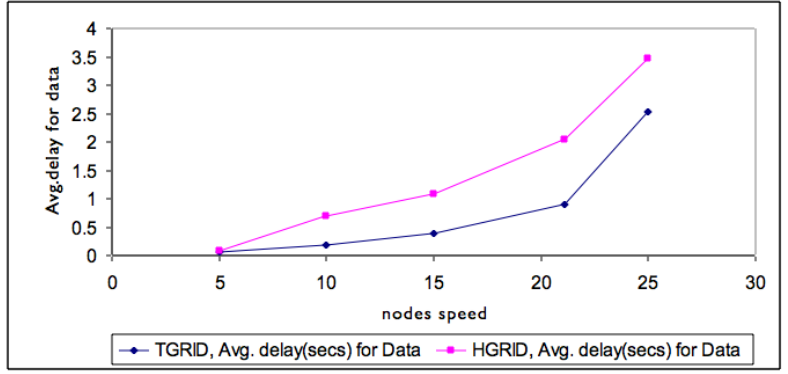

Figure 10.Delay

Figures 9 and 10 show the average data throughput and delay achieved by each protocol. Throughput decreases with mobility for both protocols, with TGRID being affected almost more by mobility. Packet delay increases with mobility. HGRID performs worse than TGRID, indicating that network congestion due to mobility causes network under-performance.

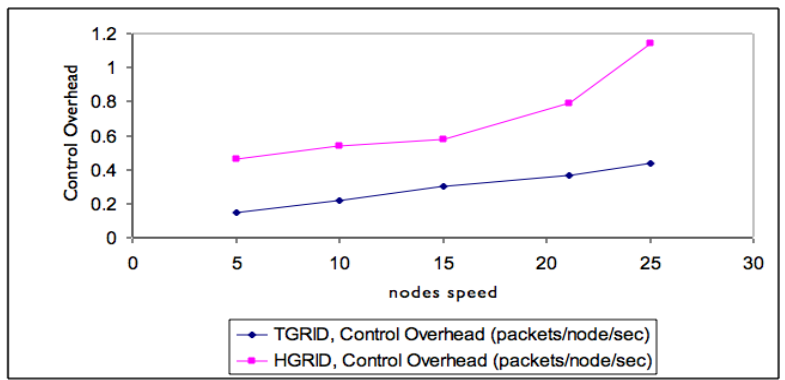

Figure 11.Control Overhead

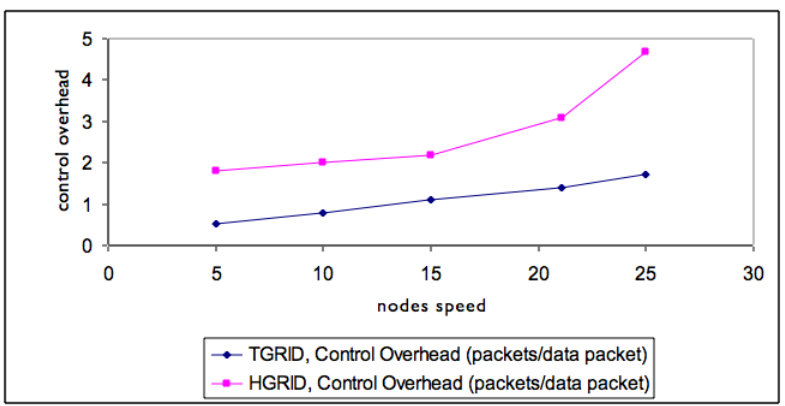

Figure 12.Control Overhead

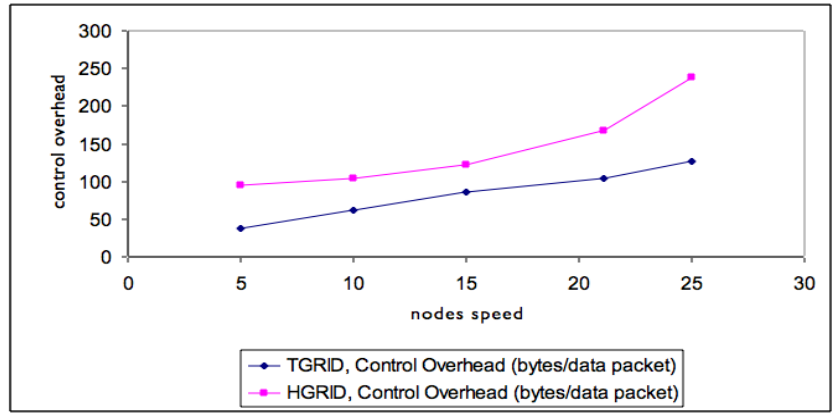

Figure 13.Control Overhead 


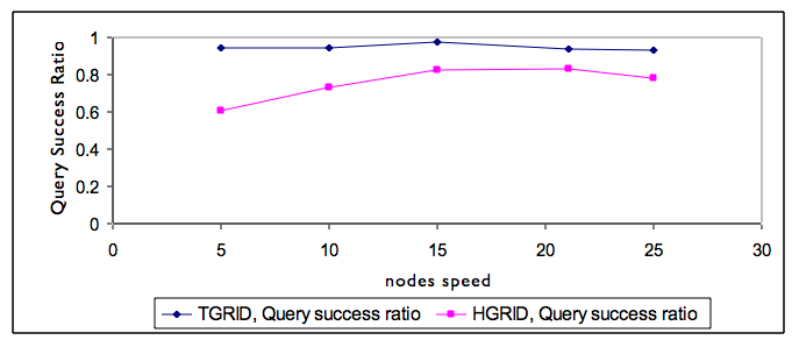

Figure 14.Query Success Ratio

In HGRID, when a node moves in highest level boundaries, it makes more updates than TGRID, so highest server grids become points of congestion and thus the bottleneck in performance as indicated by the increase in data delay with increased mobility.

Figure 14 shows the probability that a query for a destination returned successfully in TGRID is more than HGRID.

\section{CONCluSiOnS AND FUture WORKS}

We have presented TGRID as a new location service scheme for mobile ad-hoc networks. The results show that how well this scheme scales as the density of the network, denoted by the number of nodes $\mathrm{N}$, increases, and the moving speed of a node, denoted as $\mathrm{v}$, increases versus the well known scheme HGRID.

In TGRID scheme the node that is located near the middle of the whole region has the least update and query cost, and the nodes in the middle square know the relative location of any other nodes in the network. It requires large storage in middle nodes but it can be solved by just taking 3 bits for each node and hash each node's ID to the stored table that each row just have 3 bits. These 3 bits show the next higher level location server, and must select one of the 8 location server zones that are around.

In terms of the total overheads, TGRID overcomes HGRID. We point out that if nodes distributed normally, TGRID performs in its best case.

Finally we indicate that scalability of location management scheme can affect the scalability of location-based routing.

The research about the TGRID Location Service is by far not complete. The location service in its base version needs to be studied further by simulating it under different scenarios.

\section{REFERENCES}

[1] J. Li, "A Scalable Location Service for Geographic Ad Hoc Routing," MobiCom 2000, Proceedings of the Sixth Annual International Conference on Mobile Computing and Networking, pp. 120-30, 2000.

[2] Y. Yu, G. H. Lu, Z. Li. Zhang, "Location Service in Ad-Hoc Networks: Modeling and Analysis," In Proceeding of NSF Workshop on Theoretical and Algorithm Aspect of Ad Hoc Wireless Networks, Chicago, June 2004.

[3] S.J.Philip, J.Ghosh, "Scalability Analysis of Location Management Protocols for Mobile Ad Hoc Networks," in Proceeding of IEEE WCNC('04), Atlanta Georgia, USA, Mar. 2004.

[4] S. M. Das, H. Pucha and Y. Charlie Hu, "Performance Comparison of Scalable Location Services for Geographic Ad Hoc Routing," INFOCOM 2005, 24th Annual Joint Conference of the IEEE Computer and Communications Societies, pp. 1228-1239, March 2005. 
[5] I. Stojmenovic, "Position Based Routing in Ad hoc Networks," IEEE Communications Magazine, vol.40, no 7, pp. 128-134, July 2002.

[6] T. Camp, J. Boleng, and L. Wilcox, "Location information Services in Mobile Ad-hoc Networks," in Proceedings of the IEEE International Conference on Communications (ICC), pp. 3318-3324, 2002.

[7] S.J. Philip, C. Qiao, "Hierarchical Grid Location Management for Large Wireless Ad hoc Networks," in Proceedings of ACM MobiHoc 03, June 2003.

[8] GloMoSim: Global Mobile Information Simulation Library. http://pcl.cs.ucla.edu/projects/glomosim/.

[9] M. Mauve, H. Hartenstein, "A Survey on Position-Based Routing in Mobile Ad-Hoc Networks," IEEE Network, vol.15, no. 6, pp. 30-39, December 2001.

[10] Liu, Jianqi, Jiafu Wan, Qinruo Wang, Pan Deng, Keliang Zhou, and YupengQiao. "A survey on position-based routing for vehicular ad hoc networks." Telecommunication Systems 62, no. 1 (2016): $15-30$.

[11] Borghei, Hooman. "Ad hoc formation and tracking of location-sharing groups." U.S. Patent 9,369,847, issued June 14, 2016.

[12] Sarkar, Subir Kumar, TipturGangarajuBasavaraju, and C. Puttamadappa. Ad hoc mobile wireless networks: principles, protocols, and applications. CRC Press, 2016.

[13] Balico LN, Loureiro AA, Nakamura EF, Barreto RS, Pazzi RW, Oliveira HA. Localisation prediction in vehicular ad hoc networks. IEEE Communications Surveys \& Tutorials. 2018 Jan 1;20(4):2784803.

[14] Martin, E., Liu, L., Covington, M., Pesti, P. and Weber, M., 2018. Positioning Technologies in Location-Based Services. In Location-Based Services Handbook (pp. 15-60). CRC Press.

[15] Chien, G. and Arasavelli, Y., AT\&T Intellectual Property I LP and AT\&T Mobility II LLC, 2019. Ad hoc group bidding. U.S. Patent Application 10/200,998.

[16] Zhang, Shaobo, Kim-Kwang Raymond Choo, Qin Liu, and Guojun Wang. "Enhancing privacy through uniform grid and caching in location-based services." Future Generation Computer Systems 86 (2018): 881-892.

[17] Lin, Jiaping, JianweiNiu, Hui Li, and Mohammed Atiquzzaman. "A Secure and Efficient Locationbased Service scheme for smart transportation." Future Generation Computer Systems 92 (2019): 694704.

[18] Cui, Jie, Jingyu Wen, Shunshun Han, and Hong Zhong. "Efficient privacy-preserving scheme for realtime location data in vehicular ad-hoc network." IEEE Internet of Things Journal 5, no. 5 (2018): 3491-3498.

[19] Martin, Eladio, Ling Liu, Michael Covington, Peter Pesti, and Matthew Weber. "Positioning technologies in location-based services." Location-Based Services Handbook-Applications, Technologies, and Security (2018): 1-45.

\section{AuThORS}

FarrokhleghaBaktashmotlagh is a Lecturer at the Technical and vocational University with a research focus, Approximation Algorithm and Ad-hoc network. She received the MS degree in computer science from the ShahidRajaee Teacher Training University in 2003. She received bachelor of telecommunication engineering from Tabriz University in 1994. Currently, she is a Education director of computer department at Technical and vocational University.

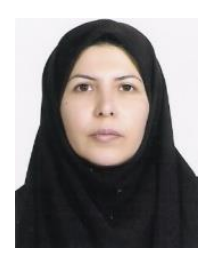

